\title{
Multivehicle Cooperative Lane Change Control Strategy for Intelligent Connected Vehicle
}

\author{
Jie Ni (iD, Jingwen Han, and Fei Dong \\ School of Automotive and Traffic Engineering, Jiangsu University, Zhenjiang 212013, China \\ Correspondence should be addressed to Jie Ni; jsdxnj@ujs.edu.cn
}

Received 3 June 2019; Revised 7 December 2019; Accepted 21 January 2020; Published 28 February 2020

Academic Editor: Hocine Imine

Copyright (C) 2020 Jie Ni et al. This is an open access article distributed under the Creative Commons Attribution License, which permits unrestricted use, distribution, and reproduction in any medium, provided the original work is properly cited.

\begin{abstract}
In order to improve the safety, stability, and efficiency of lane change operating, this paper proposes a multivehicle-coordinated strategy under the vehicle network environment. The feasibility of collaborative lane change operation is established by establishing a gain function based on the incentive model. By comparing lane change gain with lane keeping gain, whether it is feasible to perform the collaboration under current conditions can be judged. Based on the model predictive control (MPC), a multiobjective optimization control function for cooperative lane change is established to realize the distributed control. A novel two-stage cooperative lane change framework is proposed, which divides the lane change process into the lane change phase and the longitudinal headway adjustment phase. It is significant to solve the difficult numerical problem caused by the dimension of collision-avoidance constraints and the nonlinearity of vehicle kinematics. In the first stage, the subject vehicle completes lane change operation. Both longitudinal and lateral movements of the vehicle are considered to optimize the acceleration and the error of following distance at this stage; in the second stage, the operation of adjusting longitudinal headway between vehicles in the target lane is completed, and at this period, only the longitudinal motion of the vehicle is considered to optimize the vehicle acceleration error. The rolling optimization time domain algorithm is used to solve the optimization control problem step by step. Finally, based on the US NGSIM open-source traffic flow database, the accuracy and feasibility of the proposed strategy are verified.
\end{abstract}

\section{Introduction}

Lane change is one of the most basic driving behaviors with a high risk. Compared to car-following behavior, the lane change considers more vehicles, and the decision-making process is more complicated and difficult to describe. According to EU statistics, the proportion of traffic accidents caused by lane change accounts for about $5 \%$, and the traffic delay reaches $10 \%$. At the same time, $75 \%$ of the lane change accidents were caused by the driver's mistaken decisions. That is, the subject vehicle status information and the surrounding environment information were not sufficiently perceived [1]. In recent years, with the advanced sensor technology and the application of vehicle-to-vehicle (V2V) communication technology in automobiles, the interconnection between vehicles and road facilities is becoming a reality. With advanced vehicle networking technology, the acquisition of vehicle information around the driving section can provide more optimized lane change planning and speed control strategies during the vehicle running so that the microscopic traffic flow such as vehicle velocity, the headway, and the time interval of vehicles during the lane change process changes benignly in characteristics to improve the safety and comfort of vehicle driving $[2,3]$.

There have been several research studies on examining the connected vehicle environment by taking more than one vehicle into consideration in driving models. Fridman et al. [4] put forward a traffic simulation called deep traffic where the planning systems for a subset of the vehicles are handled by a neural network as part of a model-free, off-policy reinforcement learning process. It used the deep reinforcement learning method to investigate crowd-sourced hyperparameter tuning of the policy network for self-driving cars. Li et al. [5] of Beijing University of Aeronautics and 
Astronautics proposed a collaborative model of the confluence zone to further solve the security problem of the confluence zone. Li and Zhang [6] proposed a dynamical model based on the strategy of three-vehicle cooperation driving, achieving smoother acceleration distribution in the new model through considering the dynamic collaboration in the car-following condition. Hoc [7] of the French National Academy of Sciences believed that coordinated lane change was that these multiagents could take actions to resolve conflicts and accomplish personal or common goals when multiple vehicles or agents travelled on the road occurring collide. Heesen et al. [8] of the German Aerospace Centre studied the cooperative behavior that drivers may take during actual lane change. It used a test method to examine typical scenarios and performs regression processing using a logical model.

At present, the research of multivehicle cooperative lane change mainly focuses on two aspects: one is the research on the decision-making model of cooperative lane change. Ngoduy and Jia [9] proposed a continuum traffic model considering both multiple forward and backward driving strategies and point out that such driving strategy can be realized through cooperative driving strategy of connected vehicles. Li et al. [10] described the relationship among vehicles on multiple lanes by threat function and established a cooperative lane-changing rule model STCA-A based on the cellular automation model. Cao et al. [11] studied the model of cooperative lane changing in merging area of expressway and established a framework of lane-changing decision based on the driving characteristics. Nie et al. [12] proposed a decentralized cooperative lane-changing decision-making framework (DCLDF) for CAVs, which was composed of state prediction module, candidate decision generation module, and candidate decision coordination module. MenéndezRomero et al. [13] presented a novel method that automatically adapts the driving behavior, integrating the merging intention of other vehicles. The other aspect is the research on the cooperation mode and control of lane-changing vehicles. Luo et al. [14] of Nissan Motors used the model prediction control (MPC) to optimize the vehicle motion trajectory of the confluence area. The model simplified the cooperative behavior into a cooperative model of two vehicles, those are the combined vehicle and the coordinated vehicle on the original lane. At the same time, the vehicle was a mass point, and the road was a straight line. Atagoziyev et al. [15] categorized all the lane-change scenarios into seven typical cases; in each case, only one vehicle has the lane change intention; the surrounding normal vehicles cooperatively adjust the formation until the central lane-change vehicle can change the lane safely; this single-vehicle lane change process continues sequentially if more than one vehicle intends to change lane. Li et al. [16] formulated the multivehicle lane change motion planning task as a centralized optimal control problem and proposed a progressively constrained dynamic optimization (PCDO) method to facilitate the numerical solving process of this complicated problem.

A review of the prevalent studies is summarized in Table 1. As reviewed above, various decision-making and control models have been applied to the study of cooperative lane change. However, most of these research studies are based on the unmanned vehicles assumptions, the multivehicle cooperative driving system structures are designed, and the self-driving control algorithms are constructed. No study appears to integrate the decision-making and control models of cooperative lane change. The research on cooperative driving control of multivehicle especially in the lane change scene is relatively few. What is more, the studied cooperative lane change scene is relatively simple and idealized. Additionally, due to the dimension of collisionavoidance constraints and the nonlinearity of vehicle kinematics, the optimal control function is often difficult to solve.

Hence, this paper proposes a novel multivehicle coordinated safe lane change strategy based on centralized decision-making and distributed control. In order to determine when and where to perform coordinated lane change for connected vehicles, we put forward a gain function model based on the incentive model to determine whether the original traffic condition is suitable for coordination. After the feasibility judgment, we give a collaborative lane-change optimization model based on the model predictive control to obtain the expected control input of each vehicle in real time. A typical scene during the specific time segment in the NGSIM open-source traffic database is selected, and the proposed collaborative strategy is applied to simulate by MATLAB to verify the impact of this method on improving traffic efficiency, stability, and driving safety and comfort.

The contributions of this paper are as follows:

(1) A multivehicle cooperative lane change strategy is proposed with centralized decision-making and distributed control. The lane-change decision and vehicle control are integrated to build multivehicle cooperative lane-change system architecture.

(2) In the distributed control, the lane change process is divided into two stages, and an optimization model is established. The first stage is the sparse vertical spacing stage, which enables the cooperative lanechange team to move from the original form to a sparse form to avoid collision. The second stage is the lane-change stage, which meets the safety distance and reduces the constraint requirement to avoid collision. Thus, the difficulty of solving the optimal control is decreased.

(3) The model considers the active cooperation of surrounding vehicles during lane change. Compared with general lane change, it can improve the efficiency of traffic flow and provide a more comfortable driving experience for drivers.

The reminder of the paper is organized as follows. The first part introduces the system architecture and workflow for implementing coordinated lane change; the second part describes the multivehicle coordinated lane change scene and the feasibility judgment method; the control objective function of the whole collaborative lane change strategy and its solving method are introduced on the third part. The fourth part is the simulation experiment and results analysis. 
TABle 1: Comparison of different methods.

\begin{tabular}{lcccccccccc}
\hline Characteristics & \multicolumn{1}{c}{ Reference } & \multicolumn{1}{c}{} \\
& {$[9]$} & {$[10]$} & {$[11]$} & {$[12]$} & {$[13]$} & {$[14]$} & {$[15]$} & {$[16]$} & This work \\
\hline Focus on decision-making of cooperative lane change & $\checkmark$ & $\checkmark$ & $\checkmark$ & $\checkmark$ & $\checkmark$ & $x$ & $x$ & $x$ & $\checkmark$ \\
Focus on control models of cooperative lane change & $x$ & $x$ & $x$ & $x$ & $x$ & $\checkmark$ & $\checkmark$ & $\checkmark$ & $\checkmark$ \\
Lane-change manoeuvres are not limited to specified patterns & $\checkmark$ & $\checkmark$ & $x$ & $\checkmark$ & $x$ & $x$ & $x$ & $\checkmark$ & $\checkmark$ \\
Multiple vehicles can change lanes simultaneously & $x$ & $\checkmark$ & $x$ & $\checkmark$ & $x$ & $x$ & $x$ & $\checkmark$ & $\checkmark$ \\
Collision-avoidance constraints are precise & $x$ & $x$ & $x$ & $x$ & $x$ & $\checkmark$ & $x$ & $\checkmark$ & $\checkmark$ \\
Solutions are obtained in real time & $\checkmark \checkmark$ & $\checkmark$ & $x$ & $\checkmark$ & $\checkmark$ & $\checkmark$ & $\checkmark$ & $\checkmark$ & $\checkmark$ \\
\hline
\end{tabular}

The conclusions and discussion are presented in the final section.

\section{System Architecture}

In order to realize safe and efficient lane change to the target lane for subject vehicle (SV), the surrounding intelligent networked vehicles assist to complete the task by accelerating and decelerating to achieve the purpose of making full use of road resources. The architecture for achieving system cooperation is shown on the left part of Figure 1. The intelligent sensing layer is responsible for perceiving the driving state of vehicles and road environment information; The coordination layer is mainly used to formulate multivehicle cooperative lane-change driving strategies and control targets; the vehicle control layer commands the vehicle power system by the vehicle actuator, executing expectation control according to state information of the vehicle and the cooperative driving strategy. The driving speed and direction of the vehicle are controlled by the throttle opening, the wheel-cylinder pressure, and the steering wheel angle to achieve the purpose of completing the cooperative driving task.

The system workflow is shown on the right part of Figure 1; through the intelligent sensing layer, the vehicle state parameters and surrounding environment information are obtained, and then the benefit of cooperative lane change under the present traffic situation at the original time is comprehensively evaluated, and the feasibility of coordinated lane change is centrally judged. According to the lanechange strategy proposed by the coordination layer, the expected control input amount of each vehicle is obtained, and control information is shared with each coordinated vehicle based on dedicated short-range communication (DSRC) technology. Finally, the coordinated operation is performed, and the vehicles are notified to stop the cooperation after completing this task.

\section{Application Scenarios}

3.1. Introduction to the Scene. The multivehicle coordinated lane-change scenario studied in this paper is the highway environment under the network condition shown in Figure 2. The subject vehicle (SV) changes lanes from the original lane to the target lane during the lane change process. The ALV and AFV vehicles, respectively, represent the front and rear vehicles in the target lane, and the LV vehicle represents the preceding vehicle in the original lane.
SV changes lanes from the original lane to the place between the front and rear vehicle in the target lane. During this process, information exchange among vehicles is realized through the DSRC technology. The obtained vehicle state parameters take the subject vehicle (SV) for reference. The goal of coordinated lane change is to assist in performing lane change operation safely and efficiently.

3.2. Feasibility Judgment of Lane Change. Since the lane change behavior affects the upstream and downstream vehicles of the original and target lane, it is necessary to accommodate information from a plurality of surrounding vehicles for feasibility judgment of the collaboration. In order to model the decision-making behavior of coordinated lane change, the gain function is constructed based on the incentive-based model [12]. The decision-making model formula for a lane-change vehicle (SV) is defined as follows:

$$
\begin{aligned}
& G(\mathrm{SV}, O, T)=\left(\bar{a}_{\mathrm{SV}}-a_{\mathrm{SV}}\right)+\eta\left(\min \left(\left\{\bar{a}_{t}-a_{t} \mid t \in N_{t}\right\}\right)\right) \\
& +\mu\left(\max \left(\left\{\bar{a}_{o}-a_{o} \mid o \in N_{o}\right\}\right)\right), \\
& \left\{\begin{array}{l}
N_{i}=\left\{j \in V_{i}: 0 \leq\left\|x_{\mathrm{SV}}-x_{j}\right\| \leq l\right\}, i=\{O, T\}, \\
Q_{i}=\arg \max (\mathrm{SV}, O, T),
\end{array}\right.
\end{aligned}
$$

where $\Delta a_{\mathrm{th}}$ is the switching threshold, that is, the lane change behavior is better than the lane keeping behavior under current traffic conditions. Only if the gain function $G(\mathrm{SV}, O, T)$ satisfies restriction (2), the system decision is feasible for cooperative lane change.

$$
\left\{\begin{array}{l}
G(\mathrm{SV}, O, T)>\Delta a_{\mathrm{th}}, \\
\left|\bar{a}_{\mathrm{SV}}, \bar{a}_{t}\right| \leq a_{\mathrm{safe}},
\end{array}\right.
$$

where for the subject vehicle SV, $O$ and $T$ represent the original lane and the target lane, respectively; gain function $G(\mathrm{SV}, O, T)$ indicates the overall benefit of SV switching from lane $O$ to lane $T$ in a coordinated situation; $N_{o}$ and $N_{t}$ represent subsequent vehicles in the original lane and subsequent vehicles in the target lane within the communication range $l$, respectively. $a$ shows the acceleration of the vehicle in its original state, and $\bar{a}$ presents the vehicle acceleration after lane change operating and is calculated from the state prediction model in the Section 3.3.

For the calculation of gain function $G(\mathrm{SV}, O, T)$, the first item in the right half of the formula indicates the advantage obtained by SV performing the lane change operation. In the 


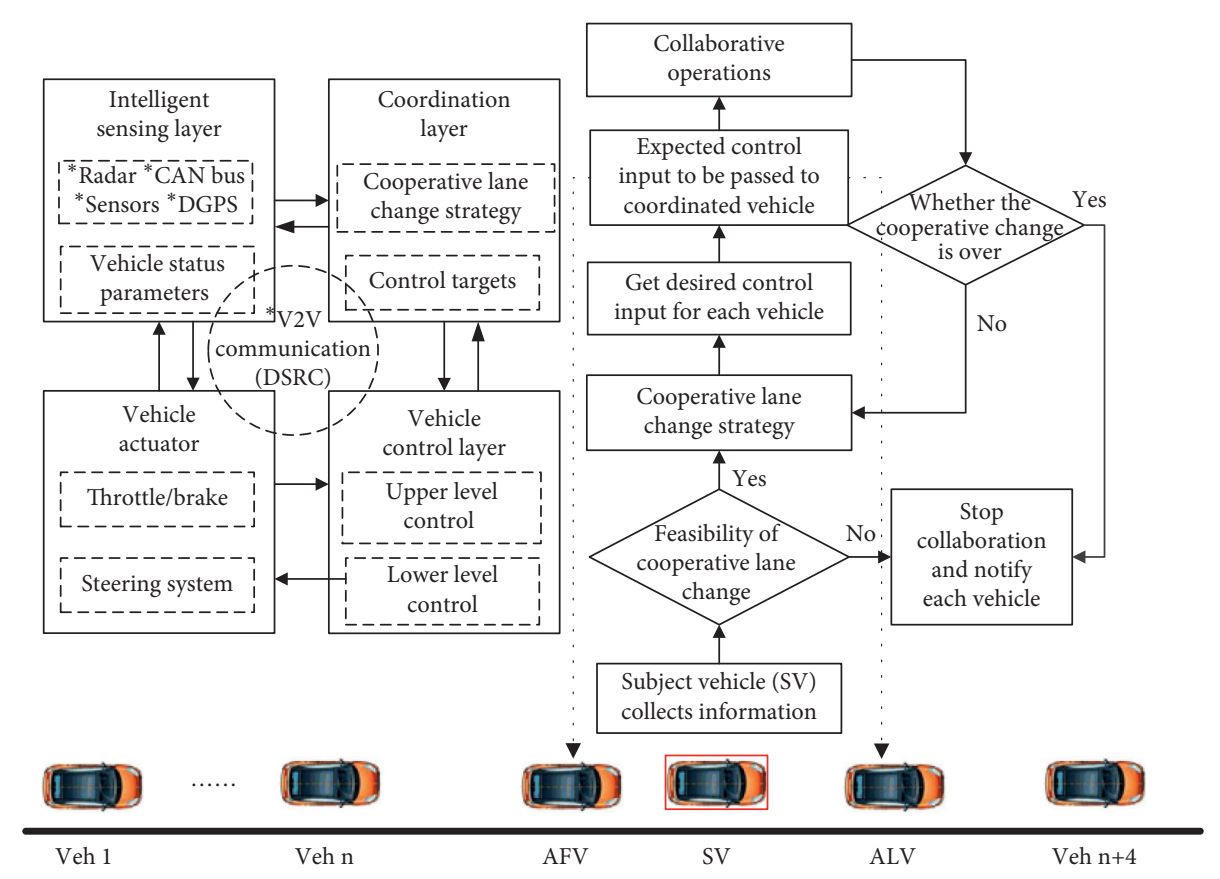

FIgURE 1: Multivehicle coordinated lane-change system architecture and workflow.

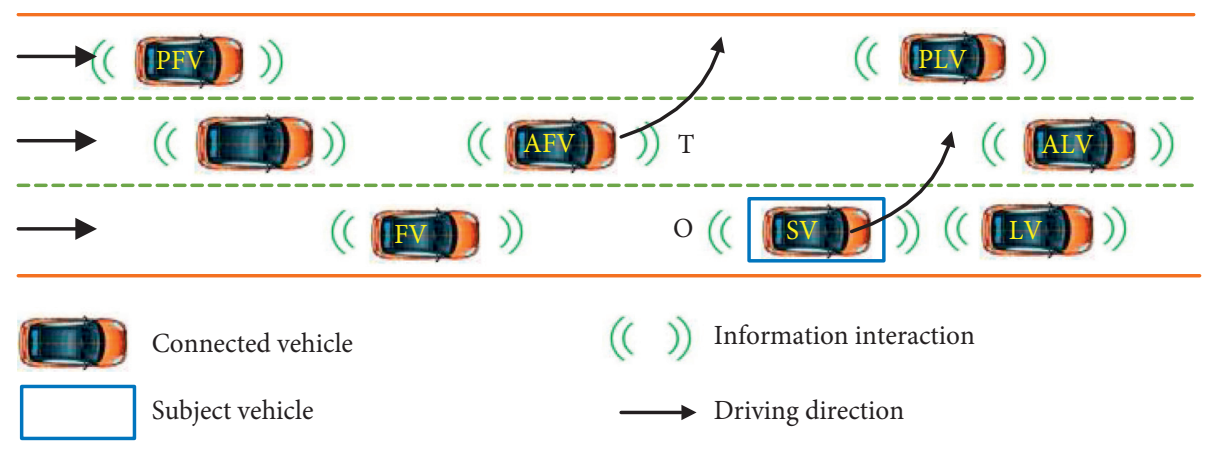

FIgUre 2: Collaborative lane change scene.

second term, $\eta$ is a courtesy factor that characterizes the impact of lane change operation on subsequent vehicles (within communication range) in the target lane, with the aim of reducing unnecessary and aggressive lane change behavior and avoiding disruption of traffic stability. Because the calculation result of $\bar{a}_{t}-a_{t}$ is always nonpositive, its minimum value represents the maximum impact of the lane change behavior. In the third item, $\mu$ is the benefit factor, which indicates the speed advantage of subsequent vehicles in the original lane due to the lane change operation and intends to encourage positive impact of the lane change behavior on traffic efficiency. The calculation result of $\bar{a}_{o}-$ $a_{o}$ is always nonnegative, so its maximum value is chosen to indicate the maximum positive impact.

3.3. State Prediction Model. In order to obtain the acceleration of each vehicle after subject vehicle (SV) changing lane, an optimal velocity model is selected as the car-following model to predict the assumed condition. The model not only reflects driver characteristics but also describes traffic flow characteristics $[17,18]$. It is shown as follows:

$$
\begin{aligned}
a_{n} & =K\left[V_{m}\left(s_{n+1}-s_{n}\right)-v_{n}\right], \\
V_{m}\left(\Delta s_{n}\right) & =V_{1}+V_{2} \tan h\left[C_{1}\left(\Delta s_{n}-l_{c}\right)-C_{2}\right],
\end{aligned}
$$

where $a_{n}$ represents the longitudinal acceleration, $\Delta s_{n}=s_{n+1}-s_{n}$ is the headway of two vehicles, $V_{m}\left(\Delta s_{n}\right)$ is the optimal velocity function, $l_{c}$ is the minimum safe distance including vehicle body length, $V_{1}$ and $V_{2}$ are constant parameters, and $C_{1}$ and $C_{2}$ are the corresponding coefficients. These four parameters all need to be calibrated. The values of each parameter can be referred to the data in previous studies [18].

\section{Modelling of Cooperative Lane Change}

From the original lane to the target lane of lane change process, the subject vehicle (SV) has both vertical and horizontal movements, and the surrounding coordinated 
vehicles adjust their longitudinal motion according to the proposed strategy [19]. In order to ensure smooth operation of the lane change operation and consider the safety and comfort, the multiobjective coordinated optimization control problem is established. In order to reduce the difficulty of solving the optimal control problem, the entire lane change process is divided into two stages. In the first stage (lane change stage), the subject vehicle starts to perform lane change and ends in entering the target lane. In the second stage (adjustment stage), the subject vehicles in the target lane and the spacing between the front and rear vehicles are adjusted. The lane change process is shown in Figure 3.

4.1. Lane Change Stage. In the lane change stage of SV, longitudinal acceleration varies with the desired control input, while lateral acceleration can be expressed by the sinusoidal function as shown in the following equation [20]:

$$
a_{y}(t)= \begin{cases}\frac{2 \pi W}{T^{2}} \sin \left(\frac{2 \pi}{T} t\right), & 0 \leq t \leq T, \\ 0, & \text { otherwise. }\end{cases}
$$

The duration of lateral movement of the entire lane change process is $T$. The lateral displacement that needs to be completed is one road width $W$. The lateral displacement can be obtained by integrating the lateral acceleration $a_{y}(t)$ twice:

$$
y(t)= \begin{cases}\frac{-W}{2 \pi} \sin \left(\frac{2 \pi}{T} t\right), & 0 \leq t \leq T, \\ 0, & t>T .\end{cases}
$$

When lateral displacement of the lane change vehicle reaches a road width, it can be regarded as completing the lane change phase, that is, $T$ stage 1 shown in Figure 3.

In order to achieve a smooth transition of the lane change process and ensure safety at the same time, the objective function defined in the lane change phase and the constraint conditions are shown in equation (6),

\subsubsection{Stage 1.}

$$
\begin{aligned}
\min _{1} U(k)= & \sum_{i=1}^{N}\left[\sum_{\beta} \lambda_{\beta} \alpha_{\beta}^{2}(k+i+1 \mid k)\right. \\
& \left.+\sum_{\varphi} h_{\varphi} \Delta_{h_{\varphi}}^{2}(k+i+1 \mid k)\right],
\end{aligned}
$$

where $U(k)$ represents the sum of comfort and tracking cost, $N$ represents the predicted time domain, $\beta=\{S \mathrm{SV}$, $\mathrm{LV}, \mathrm{ALV}, \mathrm{AFV}\}, \varphi=\{\mathrm{SV}, \mathrm{AFV}\}$, and $\lambda_{\beta}$ and $h_{\varphi}$ are the weighting factor of each item. $(k+i+1 \mid k)$ indicates that the value of time $k+i+1$ is predicted based on the information of time $k$.

In the objective function, the first term represents the acceleration optimization of the subject vehicle and the

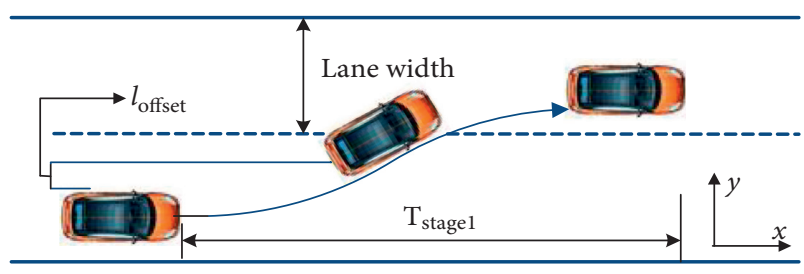

FIgURE 3: SV lane change process.

surrounding vehicles and characterizes the comfort cost in the course of lane change stage; the second term represents the following distance error between the subject vehicle and its surrounding vehicles, characterizing its tracking cost.

The constraints are as follows:

$$
\begin{gathered}
a_{s \min } \leq a_{\mathrm{SV}} \leq a_{s \max }, \\
a_{\min } \leq a_{\mathrm{LV}} \leq a_{\max }, \\
a_{\min } \leq a_{\mathrm{ALV}} \leq a_{\max }, \\
a_{\min } \leq a_{\mathrm{AFV}} \leq 0, \\
\left\|a_{\mathrm{SV}}^{\prime}, a_{\mathrm{LV}}^{\prime}, a_{\mathrm{ALV}}^{\prime}, a_{\mathrm{AFV}}^{\prime}\right\|, \\
D_{*}(k) \geq D_{\mathrm{safe}}=\max \left(\mathrm{THW} \cdot V_{f}+S_{f}, \mathrm{TTC} \cdot\left(V_{f}-V\right)+S_{f}\right),
\end{gathered}
$$

where $a_{s \min }$ and $a_{s \max }$ are the minimum and maximum comfortable lane change acceleration acceptable to the driver; $a_{\min }$ and $a_{\max }$ are the minimum and maximum comfort acceleration acceptable to the driver. $T_{p}$ is the maximum comfortable longitudinal acceleration rate acceptable to the driver. A sharp change in acceleration can also cause discomfort to the driver. (7a)-(7d) guarantee the safety and comfort of each vehicle during the lane change stage, ensuring that the state control variables are within the allowable range. (7c) indicates that the rear vehicle in the target lane cannot be accelerated. (7f) means that the distance between two workshops should not be less than the minimum safe distance. $D_{*}(k)$ indicates the actual distance between two vehicles represented by $*$ at $k$ time. THW is the safety critical follow-up time interval of two workshops, and TTC is the safety critical collision time. $V_{f}$ is the speed of the front car, $S_{f}$ is the minimum safety spacing between two vehicles.

4.2. Adjustment Stage. During the adjustment stage, the subject vehicle completes lane change operation and enters the target lane, adjusting the distance between the front and rear vehicles in the target lane. Compared with the lane change phase, this stage does not consider the front vehicle in the original lane, so constraints become sparse. The objective function and the constraint definition are shown in equation (8). 
4.2.1. Stage 2.

$$
\min _{2} U(k)=\sum_{i=1}^{N}\left[\sum_{\mu} \omega_{\mu} \Delta a_{\mu}^{2}(k+i+1 \mid k)\right],
$$

where $U(k)$ represents the comfort cost. The objective function expresses the acceleration error optimization of the self-vehicle and front and rear vehicles in the target lane to ensure its longitudinal driving comfort. $\mu=\{\mathrm{SV}, \mathrm{ALV}, \mathrm{AFV}\}$ and $\omega_{\mu}$ is the weight factor.

The constraints are as follows:

$$
\begin{gathered}
a_{\mathrm{min}} \leq \bar{a}_{\mathrm{SV}} \leq a_{\mathrm{max}}, \\
a_{\mathrm{min}} \leq \bar{a}_{\mathrm{ALV}} \leq a_{\mathrm{max}}, \\
a_{\mathrm{min}} \leq \bar{a}_{\mathrm{AFV}} \leq a_{\mathrm{max}}, \\
\left\|\bar{a}_{\mathrm{SV}}^{\prime}, \bar{a}_{\mathrm{ALV}}^{\prime}, \bar{a}_{\mathrm{AFV}}^{\prime}\right\| \leq T_{p}, \\
D_{*}(k) \geq D_{\text {safe }}=\max \left(\mathrm{THW} \cdot V_{f}+S_{f}, \mathrm{TTC} \cdot\left(V_{f}-V\right)+S_{f}\right) .
\end{gathered}
$$

4.3. Model Solving Method. Since the objective function established in this paper is a multiobjective coordination optimization control problem, the rolling time domain optimization algorithm is used to solve it. As the sampling time advances, the deviation of each time period is optimized and calculated repeatedly in finite time interval of rolling [21], and the expected input of each vehicle is obtained to realize active cooperation. Within the system predicting the time domain, the constraints are all in the form of $A X \leq B$. Therefore, this kind of management method is used to soften the hard constraints, and the Dantzig-Wolfe effective set method [22] is selected to obtain the optimal control variables:

$$
X=\arg \min U .
$$

The first element of optimal control quantity $X(0)$ is extracted and entered into the vehicle model. The desired optimal throttle opening $c_{\text {thr }}^{*}(0)$ and optimal brake pedal pressure $c_{\text {brk }}^{*}(0)$ are achieved for optimal control of vehicle driving and braking. The parameters in the model are designed as shown in Table 2.

\section{Simulation Experiment Verification}

5.1. Experimental Setup and Condition. This paper uses the US NGSIM open-source traffic flow database [23] to verify the lustiness of the proposed model. The database source records the traffic flow data of typical highways in the United States by means of fixed-point video observation and extracts the motion track data of all vehicles in the video through image processing technology. The data selected in this paper are fifteen minutes (4:00 am-4:15 am) vehicle
TABle 2: Parameter design.

\begin{tabular}{lc}
\hline Parameter symbol & Parameter value \\
\hline$\lambda_{\beta}$ & {$[0.3,0.2,0.1,0.1]$} \\
$h_{\varphi}$ & {$[0.15,0.15]$} \\
$\omega_{\mu}$ & {$[0.4,0.3,0.3]$} \\
$T_{p}$ & $1.5 \mathrm{~m} / \mathrm{s}^{3}$ \\
$a_{s \min }$ & $-1 \mathrm{~m} / \mathrm{s}^{2}$ \\
$a_{s \max }$ & $1.5 \mathrm{~m} / \mathrm{s}^{2}$ \\
$a_{\min }$ & $-4 \mathrm{~m} / \mathrm{s}^{2}$ \\
$a_{\max }$ & $4 \mathrm{~m} / \mathrm{s}^{2}$ \\
$W$ & $3.75 \mathrm{~m}$ \\
\hline
\end{tabular}

trajectory data of the 2 nd and $3 r d$ lanes in the I-80Emeryville-CA expressway, as shown in Figure 4. The section is $503 \mathrm{~m}$ (about 1,650 feet) long. In order to verify the feasibility and practicability of the proposed strategy, the actual lane change scenario at the moment in the road section without external disturbance is defined as the general traditional lane change (TLC); the simulation applied to the same scenario using the multivehicle coordinated lane change strategy is defined as a coordinated lane change condition (CLC).

5.2. Results and Discussion. Figure 5 shows the vehicle trajectory in the 2nd lane during a certain period of time. During this time period, a total of 8 lane changes occurred, six lane changes were from the 3 rd lane to the 2 nd lane and two lane changes were from the 2 nd lane to the 3 rd lane. The color in the figure represented the speed of the vehicle, and the larger the color value, the greater the speed. It could be seen from Figure 5 that congestion occurred in the position 400-800 feet section from frame 480 to frame 1000, especially after two lane change behaviors, and the congestion was obvious. After applying the multivehicle coordinated lane change strategy (Figure 6), the impact caused by the lane change became smaller, and the congestion was effectively controlled. The vehicle speed was increased, the time for the vehicle to reach the same position was reduced by $3-11 \mathrm{~s}$, and the traffic efficiency was improved. The feasibility of lane change was also calculated here. There was no significant change in the overall number, but the timing of the lane change changed.

The average speed is the average value of all vehicles' speed in a certain time or space, which is the important performance indicator of the traffic system. Figure 7 shows the time average speed in each time zone in the case of TLC and CLC. Under the coordinated lane change strategy, the average time of vehicles increased from $26.96 \mathrm{~km} / \mathrm{h}$ to $30.41 \mathrm{~km} / \mathrm{h}$ in maximum. The average speed was significantly improved.

Two groups of vehicle lane changing processes were randomly selected from the above time period, and the coordinated lane changing strategy was used for simulation research. Figures 8 and 9 show the acceleration and speed curve of three vehicles during the lane change. In the traditional lane changing process (Figure 8), the overall acceleration curve of SV showed a downward trend, but there 


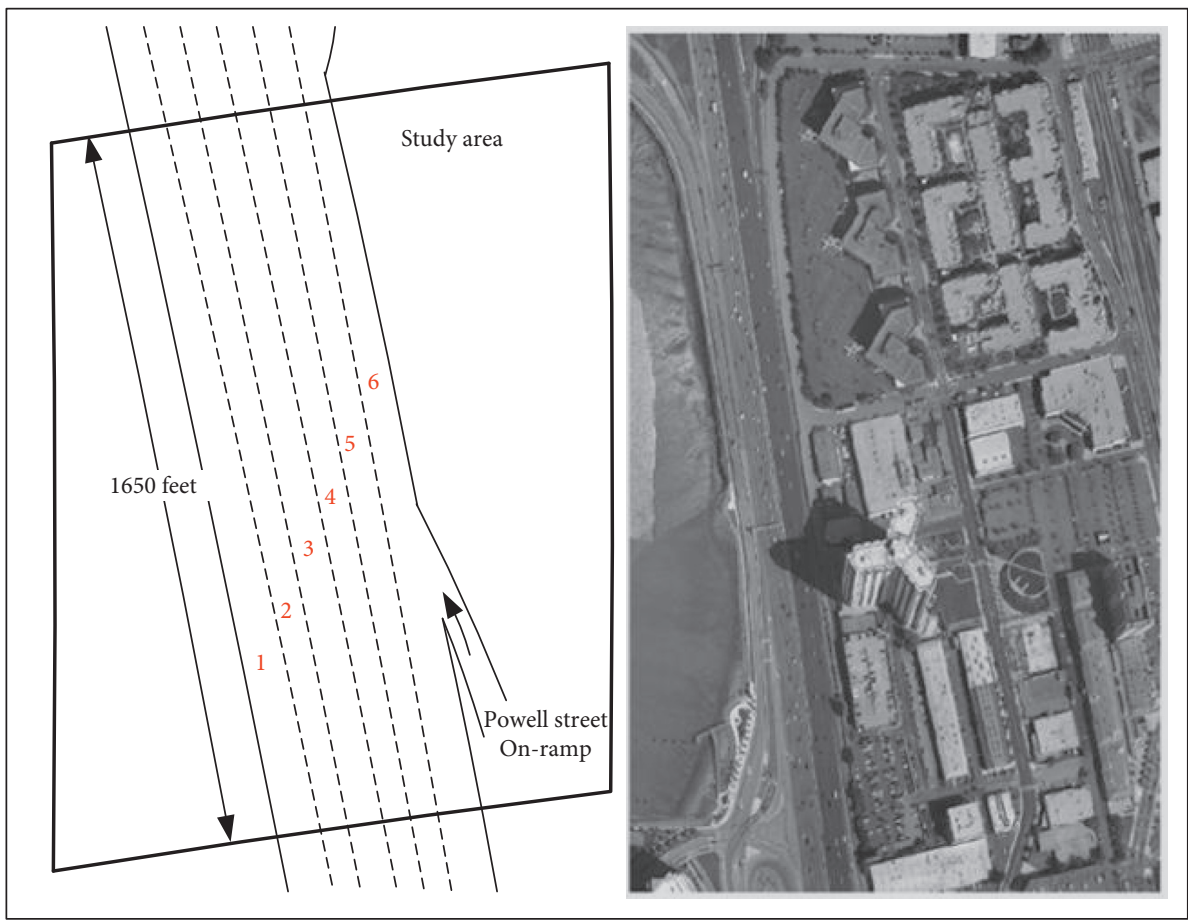

FIgURE 4: Selected highway scene.



Figure 5: Vehicle track diagram (TLC).

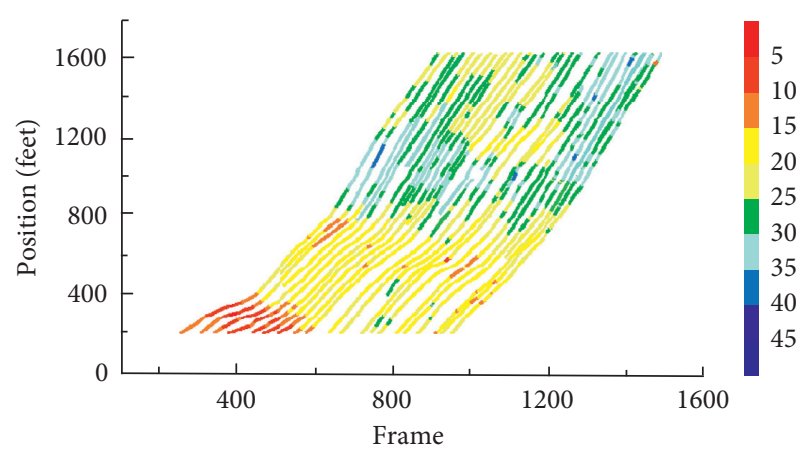

Figure 6: Vehicle track diagram (CLC).

were obvious fluctuations. After entering the target lane, the vehicle acceleration was maintained near $0 \mathrm{~m} / \mathrm{s}^{2}$, and the vehicle speed was stable. With the coordinated lane change strategy, the lane changing vehicle was always in the acceleration state during the entire lane changing process, but



Figure 7: Time average speed diagram.

the acceleration variation was small and gradually decreased. In the TLC scenario, when the vehicle in the 2 th lane was cut in, that was, in the second stage, AFV decelerated rapidly. It could be speculated reasonably that the driver generated a dangerous perception influenced by the speed of the lane change vehicle and the distance between the vehicles. The acceleration of ALV was affected by its own preceding vehicle. In this example, the safe distance between SV and ALV could be determined by setting an appropriate threshold range, and the speed change under the coordinated lane change strategy was more stable.

Figure 10 shows the comparison between the TLC trajectory and the CLC trajectory. The CLC trajectory was more continuous. The indicators reflecting the safe lane change included the time of lane change, jerk, headway of SV, and 


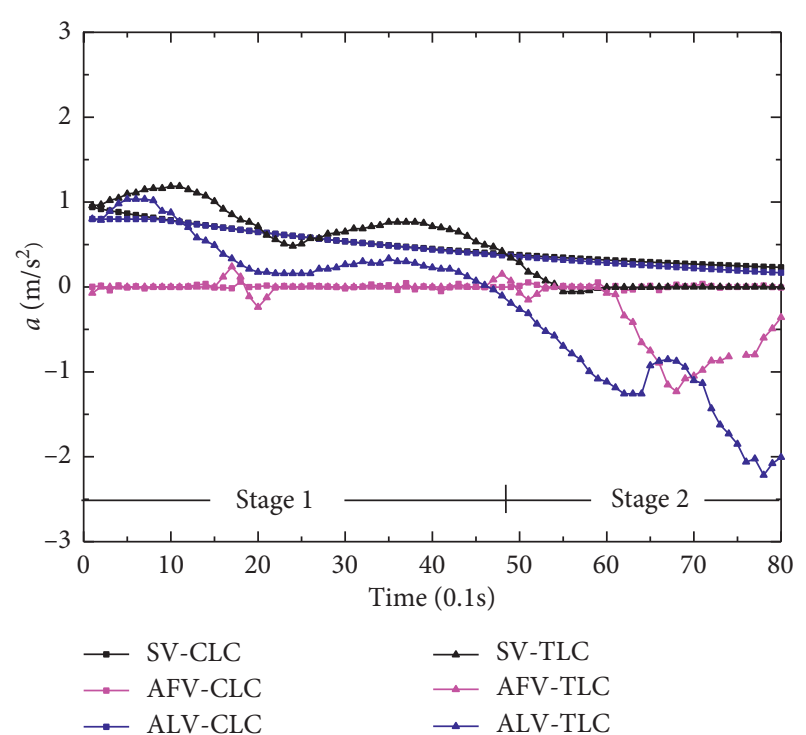

Figure 8: Vehicle accelerations during lane changing.



FIgURE 9: Vehicle speeds during lane changing.

the deceleration of ALV. Table 3 shows the comparison of these parameters in the lane change process. The time of CLC was $4.8 \mathrm{~s}$, slightly smaller than TLC, and the jerk was also significantly reduced. THW was higher than TLC, especially during the lane change process, the maximum deceleration of ALV was only $-0.09 \mathrm{~m} / \mathrm{s}^{2}$, which was significantly lower than TLC, and the safety was higher.

Similarly, Figures 11 and 12 show the acceleration and velocity curves for another scene. The speed of the target lane started to decrease significantly at the beginning in TLC, and the speed of the lane change vehicle fluctuated greatly. In CLC, SV remained stable after smooth accelerating, and the speed of AFV was basically unchanged at the whole stage.

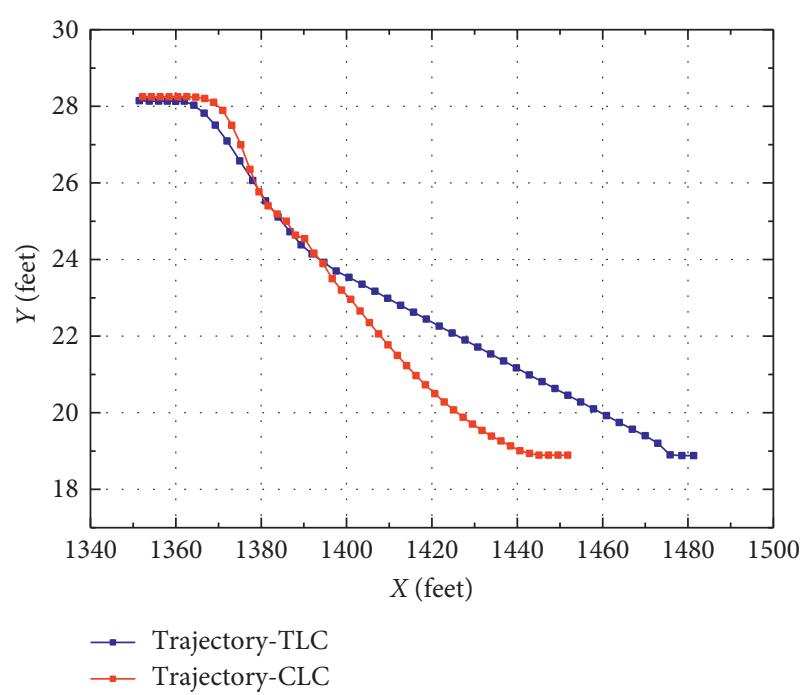

Figure 10: Vehicle trajectory of TLC and CLC.

TABLE 3: Indicator comparison of TLC and CLC.

\begin{tabular}{lcccc}
\hline Indicator & $\begin{array}{c}\text { MinTHW } \\
(\mathrm{s})\end{array}$ & $\begin{array}{c}\text { SV } \\
\text { MaxJerk } \\
\left(\mathrm{m} / \mathrm{s}^{3}\right)\end{array}$ & $\begin{array}{c}\text { MergeTime } \\
(\mathrm{s})\end{array}$ & $\begin{array}{c}\text { ALV } \\
\text { MaxDec } \\
\left(\mathrm{m} / \mathrm{s}^{2}\right)\end{array}$ \\
\hline TLC & 2.51 & 0.92 & 5.3 & -1.32 \\
CLC & 3.30 & 0.53 & 4.8 & -0.09 \\
\hline
\end{tabular}

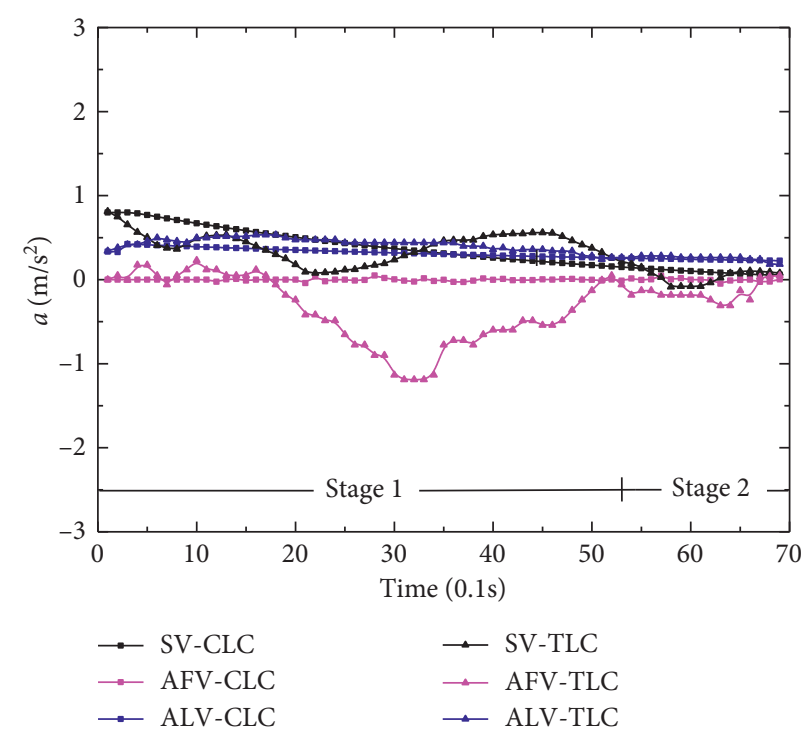

Figure 11: Vehicle accelerations during lane changing.

It could be seen from Figure 13 and Table 4 that the CLC trajectory was smooth and stable. In this scenario, the lane change time was basically unchanged, the jerk was significantly reduced, the minimum THW was increased, and the maximum deceleration of ALV was $-0.04 \mathrm{~m} / \mathrm{s}^{2}$. The effect of CLC was smaller, and the safety was even better. 


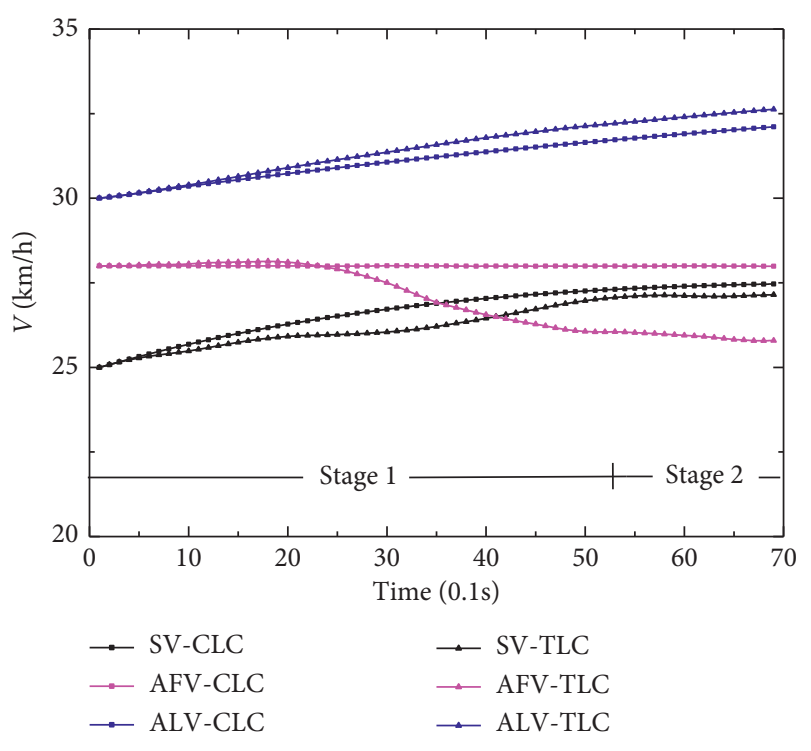

FIGURE 12: Vehicle speeds during lane changing.

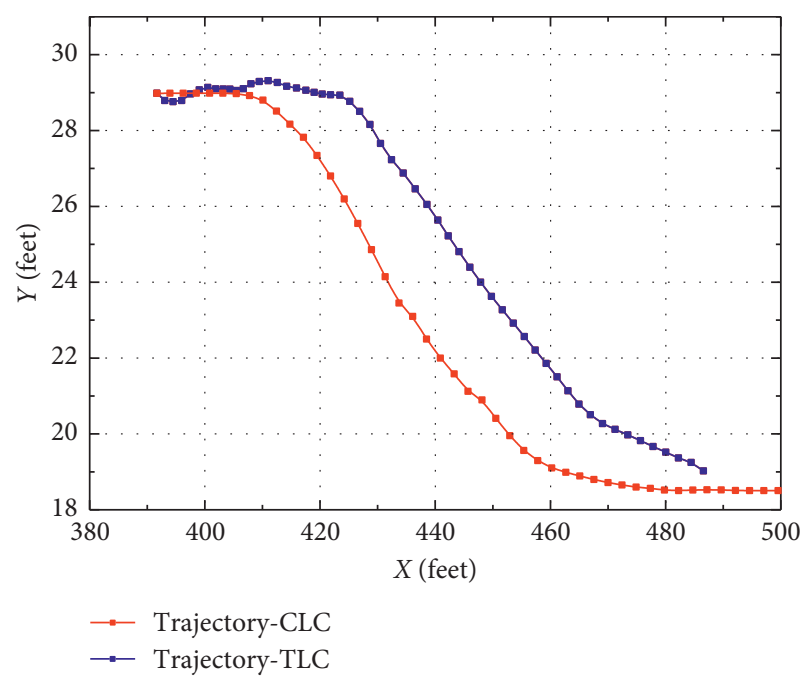

FIgURE 13: Vehicle trajectory of TLC and CLC.

TABLE 4: Indicator comparison of TLC and CLC.

\begin{tabular}{lcccc}
\hline Indicator & $\begin{array}{c}\text { MinTHW } \\
(\mathrm{s})\end{array}$ & $\begin{array}{c}\text { SV } \\
\text { MaxJerk } \\
\left(\mathrm{m} / \mathrm{s}^{3}\right)\end{array}$ & $\begin{array}{c}\text { MergeTime } \\
(\mathrm{s})\end{array}$ & $\begin{array}{c}\text { ALV } \\
\text { MaxDec } \\
\left(\mathrm{m} / \mathrm{s}^{2}\right)\end{array}$ \\
\hline TLC & 1.49 & 0.76 & 5.2 & -1.2 \\
CLC & 2.61 & 0.21 & 5.3 & -0.04 \\
\hline
\end{tabular}

\section{Conclusions}

This work is based on comprehensive real-time multivehicle information, taking active cooperation of the surrounding vehicles during lane change process into account. Compared with the general lane change, it provides drivers with a more comfortable driving experience. The multivehicle-coordinated lane change strategy based on centralized decisionmaking and distributed control divides the whole lane change process into the lane change stage and the longitudinal spacing adjustment stage, which reduce the difficulty of solving the optimal control model. The superiority of the CLC strategy over TLC is verified by the actual traffic scenarios in the NGSIM open-source traffic flow database. The results show that compared with TLC, the CLC strategy makes the lane change behavior more feasible. The deceleration of upstream vehicles will be weakened, and the shock wave in traffic flow can be relieved to some extent. During the lane change process, the acceleration of each vehicle tends to be stable, the lane change process is safer and more efficient, and the road traffic efficiency is improved.

Communication delays, system response time, and perceived errors are not considered, and this is a work that needs further improvement. In addition, in order to make the problem easy to solve, several cars in the lane change scene are all intelligent networked vehicles, which is not practical; we will further expand the influence of the system penetration and consider problems without so strong an assumption.

\section{Data Availability}

The data used to support the findings of this study have been deposited in the US NGSIM open-source traffic flow database (https://ops.fhwa.dot.gov/trafficanalysistools/ngsim. htm).

\section{Conflicts of Interest}

The authors declare that there are no conflicts of interest regarding the publication of this paper.

\section{Acknowledgments}

This research was supported by the Natural Science Foundation of the Jiangsu Higher Education Institutions (no. 17KJB580004) and Open Fund Program of the Key Laboratory for New Technologies in Vehicle Applications of Jiangsu Province (Grant no. BM20082061709).

\section{References}

[1] S. Ammoun, F. Nashashibi, and C. Laurgeau, "An analysis of the lane change manoeuvre on roads: the contribution of inter-vehicle cooperation via communication," in Proceedings of the 2007 IEEE Intelligent Vehicles Symposium, pp. 10951100, Istanbul, Turkey, June 2007.

[2] G. Yang, D. H. Zhang, K. Q. Li et al., "Parallel collaborative automatic lane change control based on vehicle and vehicle communication," Journal of Highway and Transportation Research and Development, vol. 34, no. 1, pp. 120-129, 2017.

[3] R. H. Zhang, F. You, X. N. Chu et al., "Control method of lane change in unmanned vehicles with vehicle-vehicle coordination," China Journal of Highway and Transport, vol. 31, no. 4, pp. 180-191, 2018.

[4] L. Fridman, J. Terwilliger, and B. Jenik, "Deep traffic: crowd sourced hyperparameter tuning of deep reinforcement learning systems for multi-agent dense traffic navigation," in Proceedings of the 32nd Conference on Neural Information Processing Systems (NIPS), Montréal, Canada, January 2018. 
[5] L. Li, F. Y. Wang, and H. Kim, "Cooperative driving and lane change at blind crossings," in Proceedings of the IEEE Intelligent Vehicles Symposium, pp. 435-440, IEEE, Las Vegas, NV, USA, June 2005.

[6] Z. P. Li and R. Zhang, "An extended non-lane-based optimal velocity model with dynamic collaboration," Mathematical Problems in Engineering, vol. 2013, Article ID 124908, 8 pages, 2013.

[7] J.-M. Hoc, "Towards a cognitive approach to human-machine cooperation in dynamic situations," International Journal of Human-Computer Studies, vol. 54, no. 4, pp. 509-540, 2001.

[8] M. Heesen, M. Baumann, J. Kelsch et al., "Investigation of cooperative driving behaviour during lane change in a multidriver simulation environment: human factors: a view from an integrative perspective," in Proceedings HFES Europe Chapter Conference, Toulouse, France, October 2012.

[9] D. Ngoduy and D. Jia, "Multi anticipative bidirectional macroscopic traffic model considering cooperative driving strategy," Transportmetrica B Transport Dynamics, vol. 5, no. 1, pp. 100-114, 2016.

[10] W. Li, S. R. Qu, and Y. Xia, "Coordinated lane change rules for multi-lane vehicles in a car-synchronous environment," China Highway Report, vol. 27, no. 8, pp. 97-104, 2014.

[11] W. Cao, M. Mukai, T. Kawabe, H. Nishira, and N. Fujiki, "Cooperative vehicle path generation during merging using model predictive control with real-time optimization," Control Engineering Practice, vol. 34, pp. 98-105, 2015.

[12] J. Nie, J. Zhang, W. Ding, X. Wan, X. Chen, and B. Ran, "Decentralized cooperative lane-changing decision-making for connected autonomous vehicles," IEEE Access, vol. 4, pp. 9413-9420, 2016.

[13] C. Menéndez-Romero, M. Sezer, F. Winkler, C. Dornhege, and W. Burgard, "Courtesy behavior for highly automated vehicles on highway interchanges," in Proceedings of the 2018 IEEE Intelligent Vehicles Symposium (IV), Changshu, China, June 2018.

[14] Y. Luo, Y. Xiang, K. Cao, and K. Li, "A dynamic automated lane change maneuver based on vehicle-to-vehicle communication," Transportation Research Part C: Emerging Technologies, vol. 62, pp. 87-102, 2016.

[15] M. Atagoziyev, K.-W. Schmidt, and E.-G. Schmidt, "Lane change scheduling for autonomous vehicles," IFAC-PapersOnLine, vol. 49, no. 3, pp. 61-66, 2016.

[16] B. Li, Y. Zhang, Y. Feng et al., "Balancing computation speed and quality: a decentralized motion planning method for cooperative lane changes of connected and automated vehicles," IEEE Transactions on Intelligent Vehicles, vol. 3, no. 3, 2018.

[17] L. H. Yang, S. Zhao, and H. Xu, "Research on car-following model based on improved optimized speed function," Journal of Transportation Systems Engineering and Information, vol. 17, no. 2, pp. 41-46, 2017.

[18] W. Li and W. Wang, "Complexity and applicability analysis of OVM, GFM and FVDM models," Journal of Southeast University, vol. 3, pp. 424-426, 2015.

[19] C. Katrakazas, M. Quddus, W.-H. Chen, and L. Deka, "Realtime motion planning methods for autonomous on-road driving: state-of-the-art and future research directions," Transportation Research Part C: Emerging Technologies, vol. 60, pp. 416-442, 2015.

[20] W. Li, D. Z. Gao, and J. M. Duan, "Research on free vehicle lane change model," Journal of Highway and Transportation Research and Development, vol. 27, no. 2, pp. 119-123, 2010.
[21] X. Chen, S. Zhang, L. Li, and L. Li, "Adaptive rolling smoothing with heterogeneous data for traffic state estimation and prediction," IEEE Transactions on Intelligent Transportation Systems, vol. 20, no. 4, pp. 1-12, 2018.

[22] A. Siddieg, "Implementation of the extended dantzig-wolfe method," Mathematical Theory \& Modeling, vol. 3, no. 2, pp. 12-29, 2013.

[23] V. Punzo, M. T. Borzacchiello, and B. Ciuffo, "On the assessment of vehicle trajectory data accuracy and application to the next generation simulation (NGSIM) program data," Transportation Research Part C: Emerging Technologies, vol. 19, no. 6, pp. 1243-1262, 2011. 